\title{
Hearing Aid Low Frequency Cut: Effect on Mandarin Tone and Vowel Perception in Normal-Hearing Listeners
}

\author{
Jianxing Zhang Bradley McPherson \\ Division of Speech and Hearing Sciences, Faculty of Education, University of Hong Kong, Hong Kong SAR, China
}

\section{Key Words}

Amplification $\cdot$ Chinese $\cdot$ Hearing aid $\cdot$ Mandarin $\cdot$ Noise $\cdot$ Speech intelligibility $\cdot$ Tone

\begin{abstract}
Objective: To investigate the impact of low frequency cuts (LCs) in hearing aid frequency response on Mandarin tone and vowel perception at varying signal-to-noise ratios (S/N ratios). Patients and Methods: Four LC conditions were reviewed, using a programmable, behind-the-ear hearing aid: no LC (NoLC), a 6-dB/octave LC (LC6), a 12-dB/octave LC (LC12), and an 18-dB/octave LC (LC18). Five $S / N$ ratios for speech to white noise were selected: $+10 \mathrm{~dB},+5 \mathrm{~dB}, 0 \mathrm{~dB}$, $-5 \mathrm{~dB}$ and $-10 \mathrm{~dB}$. Speech and noise stimuli were presented to 25 normal-hearing, native Mandarin-speaking listeners monaurally. Results: Tone and vowel recognition scores were high for subjects in all experimental conditions except for LC18 and S/N-10 dB. The LCs had minimal impact on tone perception in the positive $\mathrm{S} / \mathrm{N}$ and $\mathrm{S} / \mathrm{N} 0$ conditions. Significant differences for tone recognition were found between the NoLC and LC6, and the LC6 and LC18 conditions with negative $S / N$ ratios. Significant differences in vowel recognition were observed between the LC12 and LC18 settings, at all S/N conditions. Conclusion: LC hearing aid settings may negatively impact on vowel recognition, and may adversely affect Mandarin tone recognition in adverse noise conditions, in normal-hearing listeners.
\end{abstract}

Copyright @ 2008 S. Karger AG, Basel

\section{Introduction}

Modern Standard Chinese, or Mandarin, is a tonal language spoken by about 1,125 billion persons in China and in many other locations around the world [1]. Each monosyllable is pronounced with a distinctive tone, which represents a specific, corresponding lexical meaning. Tone recognition is one of the most important aspects of Mandarin speech perception [2-4]. There are four tones in Mandarin and they are usually assigned Arabic figures from tone 1 to tone 4 in sequence. To use a popular example, the monosyllable /ma/ could mean 'mother', 'linen', 'horse', or 'scold', if spoken with tone 1 to tone 4, respectively. Hence, the accurate perception of Mandarin words is dependent not only on their phonemic constituents but also on their tones.

Tonal perception depends on the perceived pitch of a speaker's voice, which is closely correlated with the fundamental frequency $\left(\mathrm{f}_{0}\right)$ of voice. Howie [5] concluded that Mandarin tones are characterized primarily by the direction of the $\mathrm{f}_{0}$ contour in the vowels. Tone 1 has a flat $\mathrm{f}_{0}$ pattern, tone 2 has a rising $\mathrm{f}_{0}$ pattern, tone 3 has a falling then rising $\mathrm{f}_{0}$ pattern, and tone 4 has a falling $\mathrm{f}_{0}$ contour. However, a number of studies have shown that in addition to the main $\mathrm{f}_{0}$ cue, other acoustic cues also support Mandarin tone perception. Liang [2] obtained high scores for Mandarin tone recognition in subjects, using high-pass-filtered Mandarin speech, indicating that tonal perception appeared to be independent of $\mathrm{f}_{0}$. This in-

Dr. Bradley McPherson, Associate Prof.

Division of Speech and Hearing Sciences, Faculty of Education

University of Hong Kong 5F, Prince Philip Dental Hospital

34 Hospital Road, Hong Kong SAR (China)

Tel. +852 2859 0592, Fax +852 2559 0060, E-Mail dbmcpher@hkucc.hku.hk 
dicated that the residual pitch of $\mathrm{f}_{0}$, its harmonic components, could cue nearly perfect performance in tonal perception. Stagray et al. [6] further explored the role of harmonics by measuring the identification thresholds of acoustically filtered Mandarin phonemes and found that higher harmonics still cued tone phonemes. These findings were consistent with predictions based on our knowledge of pitch perception for nonspeech, complex tones.

In addition to $f_{0}$ and its harmonics, there may be other acoustic cues that also inform Mandarin tone perception. Liang [2] also examined Mandarin tone perception cues using whispered speech, in which neither $\mathrm{f}_{0}$ nor its harmonic constituents were available. The study found a $64 \%$ tone recognition score with whispered speech, indicating that additional temporal envelope cues also play an important role in tone perception. More recently, studies on tone perception have adopted synthetic speech stimuli rather than natural stimuli because their acoustic parameters can be more completely specified and controlled. Whalen and $\mathrm{Xu}$ [7] reported an approximately $70 \%$ tone recognition level using signal-correlated noise stimuli, which were generated by manipulating natural speech to remove $f_{0}$ and formant structure but retain original amplitude contour and duration. Further, tones 2,3 , and 4 were found to be perceptible even when the duration cue was eliminated. Their study indicated that amplitude cues appeared to cue tone identification independently to some extent. Fu et al. [8] investigated the temporal cues in Mandarin tone perception. They obtained an about $80 \%$ tone recognition level by using a manipulated stimulus with primarily temporal envelope cues. Therefore, many cues may contribute to tonal perception in Mandarin. However, there is a consensus in the literature that $\mathrm{f}_{0}$ and its harmonics are important cues in Mandarin tone perception. Furthermore, Fu et al. [8] found that a significant relationship existed between tone and phoneme recognition, and between tone and sentence recognition. This result was consistent with the fact that Mandarin tones are lexically meaningful and indicates that damaged tone perception ability will undoubtedly reduce Mandarin speech understanding.

All the above-mentioned studies were based on normal-hearing listeners, who could utilize a rich inner redundancy system in speech perception. For a hearingimpaired person, the situation is different because they typically have poor frequency and temporal resolution, and less auditory perception redundancy due to hearing loss and, in many cases, limited language experience. However, Liu et al. [9] reported above 70\% Mandarin tone discrimination scores in mild to moderate sensorineural hearing-impaired adults in quiet conditions who were provided with sufficient amplification. No significant differences for tone recognition were noted in their study between mild sensorineural hearing loss (SNHL) listeners and a control group of normal listeners, but a significant difference was noted between moderate SNHL listeners and a control group, indicating that those who have more than mild SNHL could be more adversely affected in tone perception capability. However, little research has been conducted to evaluate the tone perception capability of hearing-impaired individuals or the possible adverse impact introduced by limitations of hearing aid frequency response.

Low frequency shaping (or low frequency cut) is a frequently used hearing aid fitting strategy to improve speech intelligibility in noise, to achieve equalized loudness across frequencies and to avoid upward spread of masking effects [10]. A low cut frequency response is often preferred by adults with SNHL [11]. For noise programs in modern digital, multiprogrammable hearing aids, the low frequency cut is often steep to decrease the impact of environmental noise and increase the consonant-to-vowel ratio. There may be a conflict in implementing low frequency shaping in hearing aid fitting for native Mandarin speakers as the electroacoustic processing of speech signals may negatively impact on tone perception by reducing or eliminating the relevant cues. In addition, harmonic cues may also be affected, depending on the cutoff frequency and roll-off slope of a hearing aid's low frequency cut. Other spectral fine structure cues, such as temporal and amplitude contours, could be smeared through hearing aid processing as well. This may be the case even for normal-hearing people, especially in competing noise conditions. The purpose of the present study was to explore the potential impact of hearing aid low frequency cut on Mandarin tone perception in various listening conditions, with normal-hearing listeners. At the same time, Mandarin vowel recognition was also considered, to examine the relationship between tone and vowel recognition and the effect of low frequency cut in hearing aid frequency response on Mandarin understanding at the phonemic detection level under various listening conditions.

\section{Methods}

The present study investigated Mandarin tone and vowel recognition for normal-hearing native Chinese speakers with two experimental treatments, a hearing aid's low frequency cut and competing noise. The low frequency cuts utilized were: no low 
Table 1. The average formant frequencies (F) in $\mathrm{Hz}$ for Mandarin vowel stimuli (tone 4), spoken by a male speaker [from ref. 5]

\begin{tabular}{lrrr}
\hline Vowel & \multicolumn{1}{c}{$\mathrm{F}_{1}$} & \multicolumn{1}{c}{$\mathrm{F}_{2}$} & \multicolumn{1}{l}{$\mathrm{F}_{3}$} \\
\hline $\mathrm{a}[\mathrm{a}]$ & 1,060 & 1,480 & 2,800 \\
$\mathrm{o}[\mathrm{o}]$ & 240 & 660 & - \\
$\mathrm{e}[\mathrm{\gamma}]$ & 300 & 1,320 & 3,060 \\
$\mathrm{i}[\mathrm{i}]$ & 300 & 2,680 & 4,140 \\
$\mathrm{u}[\mathrm{u}]$ & 260 & 620 & - \\
$\ddot{\mathrm{u}}[\mathrm{y}]$ & 280 & 2,600 & 3,200 \\
\hline
\end{tabular}

frequency cut (NoLC), a 6-dB/octave low frequency cut (LC6), a 12-dB/octave low frequency cut (LC12), and an 18-dB/octave low frequency cut (LC18), with low frequency limits (ANSI S3.222003 [12]) of $<200,355,595$, and $750 \mathrm{~Hz}$, respectively. The noise conditions were at five signal-to-noise $(\mathrm{S} / \mathrm{N})$ ratios: $+10 \mathrm{~dB},+5 \mathrm{~dB}$, $0 \mathrm{~dB},-5 \mathrm{~dB}$, and $-10 \mathrm{~dB}$. Continuous white noise was used to represent the background noise, and the $+10 \mathrm{~dB} S / \mathrm{N}$ ratio approximated a typical low noise situation in real life.

Subjects

Twenty-five normal-hearing native Mandarin-speaking subjects (15 females and 10 males) voluntarily participated in this study. The age range was from 10 to 44 years, with a mean age of 19 years (SD 8.6). A wide age range was selected to allow consideration of age effects in the analysis. All subjects had passed puretone screening at $20 \mathrm{~dB} \mathrm{HL}$ at octave frequencies from 250 to $8,000 \mathrm{~Hz}$ in each ear [13].

\section{Stimuli}

Speech material originated from the Chinese Minimal Auditory Capability Test [14]. The experimental speech material comprised 48 stimuli, which comprised six Mandarin simple vowels (a [a]; o [o]; e [r]; i [i]; u [u]; $\ddot{u}[y]$ ) and their four tones, spoken by both a male and female native Mandarin speaker and digitally recorded at the House Ear Institute, Los Angeles. Each stimulus item was selected after being judged by the first author (a native Mandarin speaker) as a clear Mandarin utterance. The average formant frequencies for these vowels (tone 4), spoken by a male speaker, are noted in table 1 .

\section{Experimental Hearing Aid}

The experimental hearing aid was a commercial, behind-theear, programmable device with flexible low frequency shaping capability (Bernafon Audioflex 110). The hearing aid was set in a linear amplification mode to inactivate all compression functions. The position of the volume control wheel was fixed in the reference test gain position according to ANSI S3.22-1996 [12] and the reserve gain was set at $0 \mathrm{~dB}$. The maximum output of the hearing aid was set to an output sound pressure level of $130 \mathrm{~dB}$ SPL. The experimental hearing aid's electroacoustic specifications are shown in table 2. A total of three low frequency filtering circuits were available in the experimental hearing aid. Each provided a 6-dB/octave filter slope independently. They were activated in sequence in the recording procedures. Thus, low frequency cuts were generated as follows: NoLC, LC6, LC12, and LC18.
Table 2. Electroacoustic characteristics of the experimental hearing aid (ANSI S3.22-1996, 2-cc coupler)

\begin{tabular}{lc}
\hline Parameter & Characteristic \\
\hline Frequency response & $200-5,800 \mathrm{~Hz}$ \\
Full-on gain & $60 \mathrm{~dB}$ SPL (50 dB input) \\
Reference test gain & $48 \mathrm{~dB}$ SPL \\
Saturation sound pressure level & $130 \mathrm{~dB}$ SPL \\
Equivalent input noise & $26 \mathrm{~dB}$ \\
Total harmonic distortion & $<3 \%$ \\
\hline
\end{tabular}

The frequency response of the experimental hearing aid with the four low frequency cut settings is shown in figure 1. As shown in figure 1, the low frequency cutoff values also changed with activation of the filtering circuits.

\section{Recording Apparatus and Procedures}

The original speech and noise stimuli were played back via a computer routed to an audiometer and to a loudspeaker before processing through the experimental hearing aid in a Knowles Electronic Manikin for Acoustic Research (KEMAR). The recording apparatus were as follows: computer, audiometer, loudspeaker, hearing aid (worn in KEMAR right ear), Tygon tube and custom earmold of the KEMAR, the KEMAR manikin, Zwislocki coupler, recording microphone, preamplifier, and digital recorder. The recording was performed in a standard sound-treated booth. The KEMAR manikin was seated at the center of the booth with the right ear at the same horizontal plane as the center of the loudspeaker. The distance between the KEMAR manikin and the loudspeaker was $1 \mathrm{~m}$, at $0^{\circ}$ azimuth. A custom earmold of KEMAR's right ear was inserted into the right ear canal of the KEMAR, at a depth of $0.5 \mathrm{~cm}$. The hook of the experimental hearing aid was coupled to the custom earmold via a thick Tygon flexible tube, $2 \mathrm{~cm}$ long and $2 \mathrm{~mm}$ inner diameter. The recording microphone terminated into the Zwislocki coupler, which was routed to the right ear of the KEMAR. Then, the recording microphone was coupled to an impedance-matched preamplifier before being fed into the digital recorder. In this manner, final recordings were made of the speech and noise stimuli after processing by the experimental hearing aid in the four low frequency cut settings, in situ on the KEMAR ear.

\section{Test Procedures}

The final speech and noise stimuli were played back via two minidisk players routed through a GSI-61 audiometer and presented to the subjects through a TDH- 49 earphone, monaurally. During the experiment, subjects were seated in a conventional sound-treated booth (with ambient noise levels that met ANSI S3.1-1977 standards [15]). The experiment was a closed-set speech test, and subjects were asked to repeat the vowel and tone that she/he had just heard, even if they were unsure of the exact sound. Each speech and noise low frequency cut condition was given five times to each subject at the five different $\mathrm{S} / \mathrm{N}$ ratios. For the normal-hearing subjects, the speech stimuli were fixed at $60 \mathrm{~dB} \mathrm{HL}$ with noise intensity varying from 50 to $70 \mathrm{~dB} H L$ in 5 - $\mathrm{dB}$ steps to generate five $\mathrm{S} / \mathrm{N}$ ratios. In this way, a total of 20 speech lists, each 


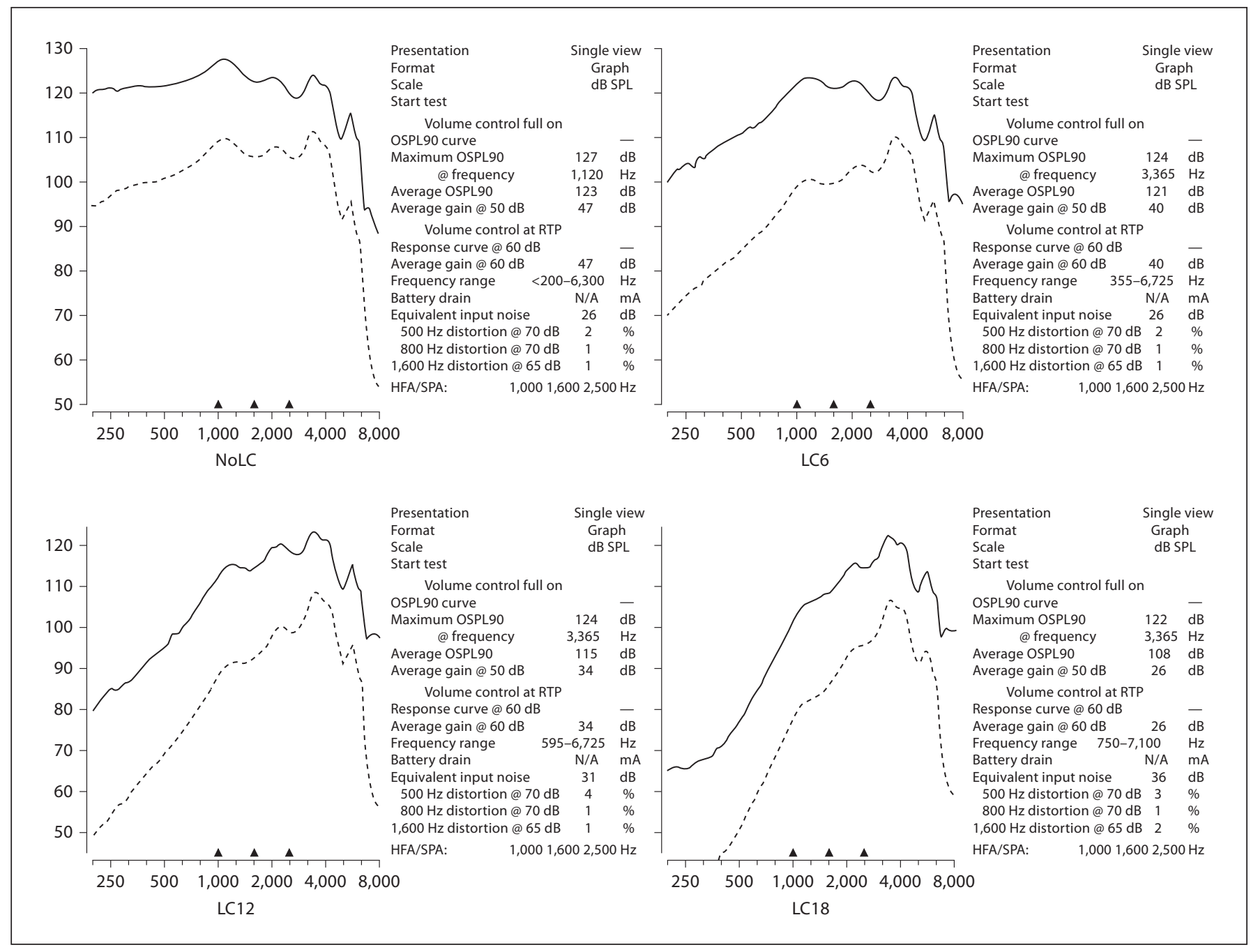

Fig. 1. Frequency response for the four low frequency cut conditions in the experimental hearing aid. The frequency response curves are shown for a $65-\mathrm{dB}$ SPL input.

of which included 48 speech items, were presented to each subject.

Standardized instructions and a pretest practice were given prior to the formal task to ensure that each subject clearly understood the test procedure. The test ear for each normal-hearing subject was selected randomly. For each speech test list, the 48 stimuli were randomly presented and the sequence of low frequency cut settings and $\mathrm{S} / \mathrm{N}$ ratios were randomly assigned to each subject.

\section{Statistical Analysis}

The Mandarin tone and vowel recognition levels were defined as the number of correctly recognized items from the total of 48 speech items in each experimental condition. The listener response was scored by the first author, a native Mandarin speaker. Parametric analysis was used to investigate the differences in speech recognition levels (tone and vowel) for the different low frequency cut settings, and to investigate speech rec- ognition levels at different $\mathrm{S} / \mathrm{N}$ ratios. Mixed between-and-within subject analysis of variance (ANOVA) was performed in both cases. Low frequency cut setting and $\mathrm{S} / \mathrm{N}$ ratio, respectively, served as within-subject variables in each ANOVA, with age and gender factors serving as between-subject variables. An alpha level of 0.05 was generally adopted for statistical significance. An alpha level of 0.01 was used in further pairwise comparisons after ANOVA.

\section{Results}

Tone and Vowel Recognition Levels

The means and two standard errors of correctly recognized tone and vowel scores for all experimental condi- 


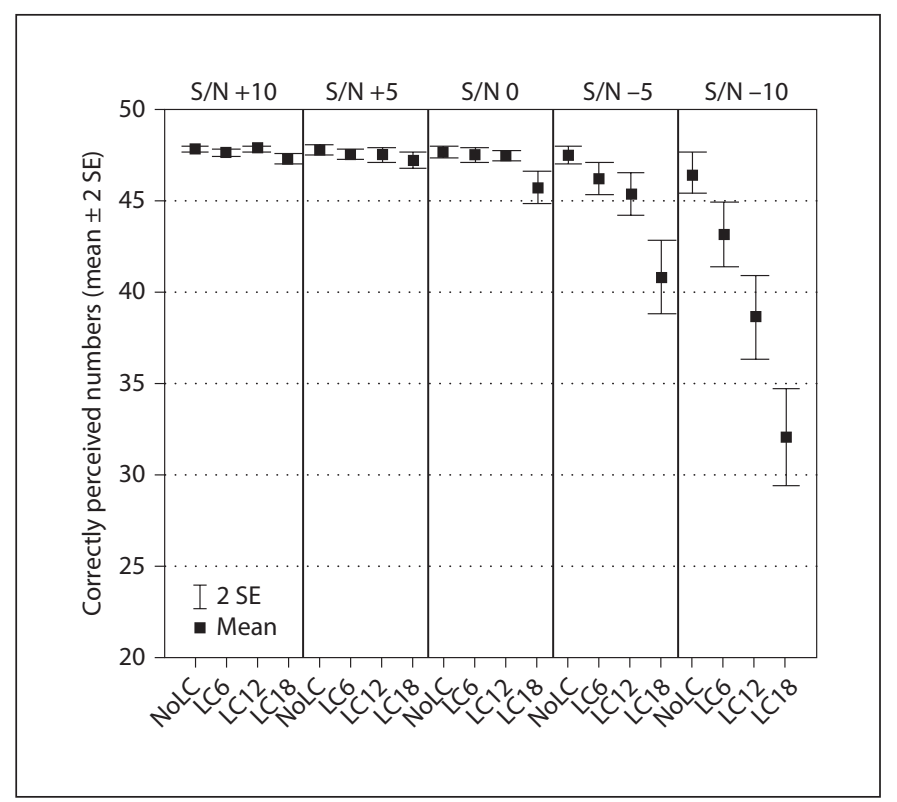

Fig. 2. The distribution of correctly perceived numbers of tones for all low cuts and $\mathrm{S} / \mathrm{N}$ ratios for normal-hearing listeners.

tions are displayed in figures 2 and 3, respectively. The overall recognition scores for both tones and vowels reached more than $90 \%$ levels except for the LC18 and $-10-\mathrm{dB} S / \mathrm{N}$ ratio conditions. Vowel recognition levels were slightly lower than tone recognition in all conditions. An obvious trend was that both tone and vowel recognition scores decreased markedly as the slope of low frequency cut increased. The LC18 setting had an adverse influence on vowel identification at all $\mathrm{S} / \mathrm{N}$ ratios. However, the low frequency cut settings only showed an influence on tone identification when $\mathrm{S} / \mathrm{N}$ ratios were negative. Neither subject age nor gender had significant interaction effects on tone or vowel recognition, in all experimental conditions.

Significant differences in tone recognition scores were noted for different low frequency cuts in the $\mathrm{S} / \mathrm{N}-5$ $[\mathrm{F}(3,9)=15.83, \mathrm{p}=0.001]$ and $\mathrm{S} / \mathrm{N}-10$ test conditions $[F(3,9)=45.82, p=0.000]$. Significant differences in tone recognition scores were noted for all $\mathrm{S} / \mathrm{N}$ ratio conditions for all low frequency cuts $[\operatorname{LC} 6 \mathrm{~F}(4,8)=35.55, \mathrm{p}=0.000$; LC12 F $(4,8)=8.71, \mathrm{p}=0.005 ; \mathrm{LC} 18 \mathrm{~F}(4,8)=17.34, \mathrm{p}=$ $0.001]$ except for the NoLC condition $[\mathrm{F}(4,8)=2.41, \mathrm{p}=$ $0.14]$.

Significant differences for vowel recognition scores were noted for different low frequency cut settings across all $\mathrm{S} / \mathrm{N}$ ratio conditions $[\mathrm{S} / \mathrm{N} 10 \mathrm{~F}(3,9)=9.11, \mathrm{p}=0.004$;

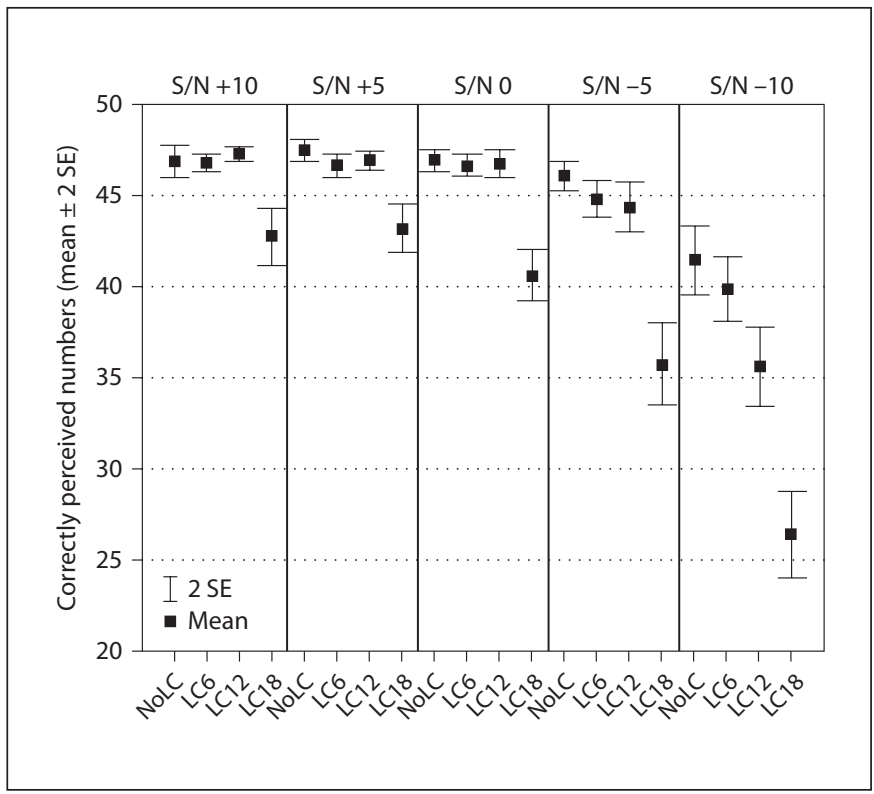

Fig. 3. The distribution of correctly perceived numbers of vowels for a variety of low frequency cuts and $\mathrm{S} / \mathrm{N}$ ratios, for normalhearing listeners.

Table 3. Pairwise comparisons of tone recognition level among all low frequency cuts in conditions $\mathrm{S} / \mathrm{N}-5$ and $\mathrm{S} / \mathrm{N}-10$, for normalhearing listeners

\begin{tabular}{|c|c|c|c|c|}
\hline \multirow[t]{2}{*}{ LCs } & \multicolumn{2}{|c|}{$\mathrm{S} / \mathrm{N}-5$} & \multicolumn{2}{|c|}{$\mathrm{S} / \mathrm{N}-10$} \\
\hline & $\mathrm{MD}$ & $\mathrm{p}$ & $\mathrm{MD}$ & $\mathrm{p}$ \\
\hline \multicolumn{5}{|l|}{ NoLC versus } \\
\hline LC6 & 1.471 & $0.000^{*}$ & 4.295 & $0.000^{*}$ \\
\hline LC12 & 2.205 & 0.044 & 7.874 & $0.000^{*}$ \\
\hline LC18 & 6.076 & $0.001^{*}$ & 13.971 & $0.000^{*}$ \\
\hline \multicolumn{5}{|l|}{ LC6 versus } \\
\hline LC12 & 0.733 & 0.481 & 3.579 & 0.013 \\
\hline LC18 & 4.605 & $0.006^{*}$ & 9.676 & $0.001^{*}$ \\
\hline LC12 versus LC18 & 3.871 & 0.034 & 6.098 & $0.009^{*}$ \\
\hline
\end{tabular}

$\mathrm{MD}=$ Mean difference. Significant difference: ${ }^{*} \mathrm{p}<0.01$.

$\mathrm{S} / \mathrm{N} 5 \mathrm{~F}(3,9)=5.17, \mathrm{p}=0.024 ; \mathrm{S} / \mathrm{N} 0 \mathrm{~F}(3,9)=14.3, \mathrm{p}=$ $0.001 ; \mathrm{S} / \mathrm{N}-5 \mathrm{~F}(3,9)=15.26, \mathrm{p}=0.001 ; \mathrm{S} / \mathrm{N}-10 \mathrm{~F}(3,9)=$ $20.38, \mathrm{p}=0.000]$. Also, significant differences in vowel recognition scores for different $\mathrm{S} / \mathrm{N}$ ratios across all low frequency cut conditions were noted $[\operatorname{NoLC} \mathrm{F}(4,8)=$ 6.22, $\mathrm{p}=0.014 ; \mathrm{LC6} F(4,8)=23.76, \mathrm{p}=0.000 ; \mathrm{LC} 12$ $\mathrm{F}(4,8)=15.31, \mathrm{p}=0.001 ; \operatorname{LC} 18 \mathrm{~F}(4,8)=16.31, \mathrm{p}=0.001]$.

Folia Phoniatr Logop 2008;60:179-187 
Table 4. Pairwise comparisons for tone recognition level among all S/N ratios in LC6, LC12 and LC18 conditions, for normal-hearing listeners

\begin{tabular}{|c|c|c|c|c|c|c|}
\hline \multirow[t]{2}{*}{$\mathrm{S} / \mathrm{N}$ ratios } & \multicolumn{2}{|l|}{ LC6 } & \multicolumn{2}{|l|}{ LC12 } & \multicolumn{2}{|l|}{ LC18 } \\
\hline & MD & $\mathrm{p}$ & MD & $\mathrm{p}$ & MD & $\mathrm{p}$ \\
\hline \multicolumn{7}{|l|}{$\mathrm{S} / \mathrm{N} 10$ versus } \\
\hline S/N 5 & 0.198 & 0.173 & 0.229 & 0.372 & 0.136 & 0.650 \\
\hline $\mathrm{S} / \mathrm{N} 0$ & 0.069 & 0.793 & 0.300 & 0.247 & 1.293 & 0.042 \\
\hline $\mathrm{S} / \mathrm{N}-5$ & 1.562 & $0.003^{*}$ & 2.464 & 0.018 & 5.943 & $0.001^{*}$ \\
\hline $\mathrm{S} / \mathrm{N}-10$ & 5.598 & $0.000^{*}$ & 9.345 & $0.000^{*}$ & 15.050 & $0.000^{*}$ \\
\hline \multicolumn{7}{|l|}{$\mathrm{S} / \mathrm{N} 5$ versus } \\
\hline $\mathrm{S} / \mathrm{N} 0$ & 0.129 & 0.535 & 0.071 & 0.850 & 1.157 & 0.051 \\
\hline $\mathrm{S} / \mathrm{N}-5$ & 1.364 & $0.009^{*}$ & 2.236 & 0.035 & 5.807 & $0.000^{*}$ \\
\hline $\mathrm{S} / \mathrm{N}-10$ & 5.400 & $0.000^{*}$ & 9.117 & $0.000^{*}$ & 14.914 & $0.000^{*}$ \\
\hline \multicolumn{7}{|l|}{$\mathrm{S} / \mathrm{N} 0$ versus } \\
\hline $\mathrm{S} / \mathrm{N}-5$ & 1.493 & $0.000^{*}$ & 2.164 & 0.026 & 4.650 & $0.003^{*}$ \\
\hline $\mathrm{S} / \mathrm{N}-10$ & 5.529 & $0.000^{*}$ & 9.045 & $0.000^{*}$ & 13.757 & $0.000^{*}$ \\
\hline $\mathrm{S} / \mathrm{N}-5$ versus $\mathrm{S} / \mathrm{N}-10$ & 4.036 & $0.000^{*}$ & 6.881 & $0.000^{*}$ & 9.107 & $0.000^{*}$ \\
\hline
\end{tabular}

$\mathrm{MD}=$ Mean difference. Significant difference: ${ }^{*} \mathrm{p}<0.01$.

Table 5. Pairwise comparisons of vowel recognition level among all low frequency cuts and all $\mathrm{S} / \mathrm{N}$ ratio conditions, for normalhearing listeners

\begin{tabular}{llllll}
\hline LCs & S/N 10 & S/N 5 & S/N 0 & S/N -5 & S/N -10 \\
\hline $\begin{array}{l}\text { NoLC versus } \\
\quad \text { LC6 }\end{array}$ & 0.564 & $0.008^{*}$ & 0.563 & 0.011 & 0.038 \\
$\quad$ LC12 & 0.693 & 0.258 & 0.893 & 0.297 & $0.006^{*}$ \\
$\quad$ LC18 & 0.019 & $0.003^{*}$ & $0.000^{*}$ & $0.000^{*}$ & $0.000^{*}$ \\
LC6 versus & & & & & \\
$\quad$ LC12 & 0.059 & 0.527 & 0.985 & 0.939 & 0.011 \\
$\quad$ LC18 & $0.008^{*}$ & 0.010 & $0.000^{*}$ & $0.001^{*}$ & $0.000^{*}$ \\
LC12 versus & & & & & \\
$\quad$ LC18 & $0.001^{*}$ & $0.004^{*}$ & $0.000^{*}$ & $0.002^{*}$ & $0.000^{*}$ \\
\hline \multicolumn{2}{l}{ Significant difference: ${ }^{*} \mathrm{p}<0.01}$. & & \\
\hline
\end{tabular}

\section{Pairwise Comparisons in Tone Recognition}

In the $\mathrm{S} / \mathrm{N}-5$ condition significant differences, using Tukey post-hoc tests of pairwise differences in means, for tone recognition score were shown in the following pairs: NoLC and LC6, NoLC and LC18, LC6 and LC18. In condition of $\mathrm{S} / \mathrm{N}-10$, significant differences were shown among all low frequency cuts except between LC6 and LC12 (table 3).

In the LC6 and LC18 conditions, significant differences in tone recognition score were shown between the neg- ative $\mathrm{S} / \mathrm{N}$ ratios and all other $\mathrm{S} / \mathrm{N}$ ratios. In the $\mathrm{LC1} 2$ condition, significant differences in tone recognition score were found between $\mathrm{S} / \mathrm{N}-10$ and all other $\mathrm{S} / \mathrm{N}$ ratios (table 4).

\section{Pairwise Comparisons in Vowel Recognition}

In the $\mathrm{S} / \mathrm{N} 10$ condition, significant differences in vowel recognition scores were found between the LC6 and LC18 settings and LC12 and LC18 settings. For the $\mathrm{S} / \mathrm{N} 5$ condition, significant differences in vowel recognition scores were found in following pairs: NoLC and LC6, NoLC and LC18, LC12 and LC18. In conditions S/N 0 and $\mathrm{S} / \mathrm{N}-5$, significant differences in vowel recognition score were shown between LC18 and other low frequency cuts. In condition $\mathrm{S} / \mathrm{N}-10$, significant differences in vowel recognition were found as follows: LC18 and other low frequency cuts, NoLC and LC12. The results for all conditions are shown in table 5.

In conditions NoLC and LC12, significant differences in vowel recognition score were shown between $\mathrm{S} / \mathrm{N}-10$ and other $\mathrm{S} / \mathrm{N}$ ratios. In condition LC6, significant differences in vowel recognition score were shown as follows: $\mathrm{S} / \mathrm{N}-10$ and other $\mathrm{S} / \mathrm{N}$ ratios, $\mathrm{S} / \mathrm{N} 10$ and $\mathrm{S} / \mathrm{N}-5$, $\mathrm{S} / \mathrm{N} 0$ and $\mathrm{S} / \mathrm{N}-5$. In condition LC18, significant differences in vowel recognition score were shown between all negative $\mathrm{S} / \mathrm{N}$ ratios and other $\mathrm{S} / \mathrm{N}$ ratios. The results for all conditions are shown in table 6. 
Table 6. Pairwise comparisons of vowel recognition level among all $\mathrm{S} / \mathrm{N}$ ratios under a variety of low frequency cut conditions, for normal-hearing listeners

\begin{tabular}{lllll}
\hline S/N ratios & NoLC & LC6 & LC12 & LC18 \\
\hline S/N 10 versus & & & & \\
S/N 5 & 0.498 & 0.846 & 0.277 & 0.708 \\
S/N 0 & 0.594 & 0.630 & 0.373 & 0.031 \\
S/N -5 & 0.151 & $0.004^{*}$ & 0.010 & $0.003^{*}$ \\
S/N -10 & $0.003^{*}$ & $0.000^{*}$ & $0.000^{*}$ & $0.000^{*}$ \\
S/N 5 versus & & & & \\
S/N 0 & 0.052 & 0.243 & 0.962 & 0.042 \\
S/N -5 & 0.025 & 0.016 & 0.062 & $0.002^{*}$ \\
S/N -10 & $0.001^{*}$ & $0.000^{*}$ & $0.000^{*}$ & $0.000^{*}$ \\
S/N 0 versus & & & & \\
S/N -5 & 0.050 & $0.001^{*}$ & 0.042 & $0.004^{*}$ \\
S/N -10 & $0.001^{*}$ & $0.000^{*}$ & $0.000^{*}$ & $0.000^{*}$ \\
S/N -5 versus S/N -10 & $0.001^{*}$ & $0.000^{*}$ & $0.000^{*}$ & $0.000^{*}$ \\
\hline
\end{tabular}

Significant difference: ${ }^{*} \mathrm{p}<0.01$.

\section{Discussion}

\section{Impact of Low Frequency Cut and Noise on Tone Recognition}

The results of this study indicated that the experimental hearing aid's low frequency cuts had no impact on Mandarin tone perception in the low noise listening conditions (i.e., S/N 10, S/N 5, and S/N 0) with normalhearing listeners. Since the $\mathrm{f}_{0}$ was cut off with steep low frequency cut, such as in LC18 condition, these findings also indicate that tone perception can be independent of $\mathrm{f}_{0}$ cues, consistent with previous reports. However, in the adverse listening conditions of $\mathrm{S} / \mathrm{N}-5$ and $\mathrm{S} / \mathrm{N}-10$, significant differences in tone recognition scores were noted for the different low frequency cut settings. It is likely that the continuous noise covered or smeared the fine spectral structures, such as amplitude and temporal contours, involved in tone perception. Consequently, a conclusion can be drawn that Mandarin tone perception cues can be divided into two groups, each of which can cue tone recognition independently. One group of cues is the frequency-domain ( $\mathrm{f}_{0}$ and harmonics) group and the other group is comprised of spectrotemporaldomain (amplitude and temporal contour) cues. It is speculated that the low frequency cut might markedly distort the frequency-domain cues of tone perception without inducing damage to spectrotemporal-domain cues.
Although low frequency cuts essentially have no impact on tone perception in low noise conditions for normal-hearing listeners, their influence on listeners with hearing impairment is still not clear. However, in noisy and reverberant acoustic conditions in real life, spectrotemporal perception tone cues could be smeared to the extent that a low frequency cut could adversely impact on Mandarin tone perception for hearing-impaired listeners. This assumption needs further investigation with subjects who have hearing impairment.

Significant differences in tone recognition scores were consistently found between NoLC and LC6, and between LC6 and LC18, whereas no significant differences were noted between LC6 and LC12. A significant difference between $\mathrm{LC} 12$ and LC18 was also noted for the S/N -10 condition. This result is not surprising because the cutoff frequencies of the low frequency cuts were not evenly distributed in the frequency band in the experimental hearing aid (fig. 1). The greatest variance was between NoLC and LC6, with the other settings having smaller differences in frequency response. The significant difference between $\mathrm{LC} 12$ and $\mathrm{LC} 18$ in $\mathrm{S} / \mathrm{N}-10$ condition indicates that a noise level exceeding $10 \mathrm{~dB}$ above the speech level could seriously mask not only the spectral fine structures of the speech stimuli but also the temporal cues to some extent. However, it appears that the harmonics were more resistant to noise interference than amplitude and temporal contours. On average, greater than $80 \%$ tone recognition levels were achieved for almost all low frequency cuts and S/N ratios, except for condition LC18 and S/N -10 . A $66.8 \%$ recognition level was reached in LC18 and $\mathrm{S} / \mathrm{N}-10$, indicating that harmonic cues might play as important a role in such adverse listening conditions for tone perception, as they do in quiet [6]. In brief, a tone recognition score above $80 \%$ was maintained regardless of the influence of low frequency cuts and noise for normal-hearing listeners, except for the very adverse listening condition $\mathrm{LC} 18$ and $\mathrm{S} / \mathrm{N}-10$. The results indicate that neither low frequency cut nor noise have significant adverse effect on tone perception for normal-hearing listeners in quiet listening conditions but have considerable negative effects in noisy environments.

It was not surprising to note that tone recognition scores declined with reduced $\mathrm{S} / \mathrm{N}$ ratio. While the NoLC condition preserved a robust $\mathrm{f}_{0}$ tone perception cue, $\mathrm{f}_{0}$ contours were cut off in part or entirely in other conditions. The pairwise comparisons showed that no significant differences for tone recognition with changing low frequency cut parameters were found among the $S / N 10$, $\mathrm{S} / \mathrm{N} 5$, and S/N 0 conditions. However, consistently sig-

Folia Phoniatr Logop 2008;60:179-187 
nificant differences were noted among $\mathrm{S} / \mathrm{N} 0, \mathrm{~S} / \mathrm{N}-5$, and $\mathrm{S} / \mathrm{N}-10$ conditions, except for $\mathrm{S} / \mathrm{N} 0$ and $\mathrm{S} / \mathrm{N}-5$ in the LC12 condition. These results suggest that noise can affect Mandarin tone perception only when the noise level exceeds the speech level and causes smearing of amplitude, temporal and spectral cues, since significant reductions in scores were found only among the negative $\mathrm{S} / \mathrm{N}$ ratio conditions. The obvious reduction of tone recognition score (to $66.8 \%$ correct) in the combined LC18 and $\mathrm{S} / \mathrm{N}-10$ condition indicates that low frequency shaping with a cutoff frequency at about $500 \mathrm{~Hz}$ could begin to distort the key harmonic cues of tone perception.

In summary, these results revealed that in a typical low noise situation with a positive $\mathrm{S} / \mathrm{N}$ ratio, tone perception was not affected by noise for normal-hearing Mandarin listeners. Nevertheless, this might not be the case for listeners with hearing loss, with one reason being that poor frequency resolution could reduce the roles of $\mathrm{f}_{0}$ and harmonics in tone perception, and another being that the amplitude and temporal contours could be more easily eliminated by noise, in conjunction with the poor spectrotemporal resolution of the hearing-impaired. Thus, not only the noise but also the low frequency cut may have a significant impact on tone perception for persons with hearing impairment even in a favorable listening condition with a positive $\mathrm{S} / \mathrm{N}$ ratio. Individuals with hearing impairment who use cochlear implants have very limited access to low frequency information and are consistently noted to have poor scores for tone identification tasks $[16,17]$.

\section{Impact of Low Frequency Cut on Vowel Recognition}

At all $\mathrm{S} / \mathrm{N}$ ratios, differences in vowel recognition score were found with different low frequency cut settings. It is noteworthy that no significant tone recognition difference was found in conditions of positive $\mathrm{S} / \mathrm{N}$ ratios and $\mathrm{S} / \mathrm{N} 0$, indicating that the low frequency cuts appear to have more impact on vowel recognition than on tone recognition, especially in low noise listening conditions such as the $\mathrm{S} / \mathrm{N} 10$ condition. Formant transitions and formant frequencies play an important role in vowel recognition, especially the first three formant cues [18]. It is reasonable to speculate that the hearing aid's low frequency cuts could distort the primary formant transition cues, especially the first two key formants for most Mandarin simple vowels except [a], so as to adversely impact on vowel perception. The pairwise comparisons revealed consistently significant differences for vowel recognition between $\mathrm{LC} 12$ and LC18, at all S/N ratios. This result indicates that a low frequency shaping with a cutoff frequency at $500 \mathrm{~Hz}$ and an $18 \mathrm{~dB} /$ octave filter slope could signifi- cantly affect Mandarin vowel recognition in all listening conditions. In a sense, $500 \mathrm{~Hz}$ as a cutoff frequency for low frequency shaping might be looked upon as a 'guard line'. Damage to vowel perception may occur if intruding over it, by inducing distortion in the primary and higher formant perception cues. This result may also have practical meaning for hearing aid fitting strategies, especially as a significant difference between LC12 and LC18 was noted even in the low noise listening condition of S/N 10. On average, nearly perfect (greater than $90 \%$ correct) vowel recognition levels were achieved for all low frequency cut and $\mathrm{S} / \mathrm{N}$ ratio combinations, except for the $\mathrm{LC} 18$ and $\mathrm{S} / \mathrm{N}$ -10 conditions. The lowest vowel recognition level (55\% correct) was obtained in the combined LC18 and S/N -10 condition. These effects are likely to be more dramatic in listeners with hearing loss, who often rely to a larger extent than normal listeners on low frequency cues.

\section{Conclusion}

This study suggests that providing adequate low frequency information will improve speech perceptual cues at the tonal and phonemic detection levels for native Mandarin-speaking listeners. The results indicate that a steep low frequency cut may damage Mandarin tone and vowel perception even in normal-hearing listeners, particularly in noisy conditions. The effects of low frequency cuts on Mandarin listeners who use hearing aids are not known. A commonly accepted opinion in hearing aid fitting is that a low frequency cut can increase consonant perception by improving consonant-to-vowel ratio and reducing the upward spread of masking. However, based on the present findings in normal-hearing listeners, tone perception in Mandarin-speaking hearing aid users may be disadvantaged by this fitting strategy. Further investigation of tone perception in Mandarin-speaking individuals with hearing impairment is needed to fully resolve these issues.

\section{Acknowledgments}

We wish to express our gratitude to Bernafon AG, Switzerland, for the experimental hearing instruments used in this project, and for providing valuable guidance in their use; and to Dr. QianJie $\mathrm{Fu}$ of the House Ear Institute, Los Angeles, for his generous assistance in offering the original recorded Mandarin speech material. In addition, we are grateful to Dr. Valter Ciocca and Dr. Lena Wong, the University of Hong Kong, for their comments on an earlier draft of this report. 


\section{References}

1 Campbell GL: Compendium of the World's Languages. Vol I: Abaza to Kurdish, ed 2. London, Routledge, 2000.

2 Liang ZA: The auditory perception of Mandarin tones. Acta Physiol Sinica 1963;26:8591.

3 Lin MC: The acoustic characteristics and perceptual cues of tones in standard Chinese. Yuwen xuexi (Chinese Language Learning) 1988;204:182-193.

4 Wang RH: Speech signal processing; in Chen YB, et al (eds): Chinese Phonetics. Hefei, University of Science and Technology of China Press, 1989, pp 37-64.

5 Howie JM: Acoustical Studies of Mandarin Vowels and Tones. Cambridge, Cambridge University Press, 1976.

6 Stagray JR, Downs D, Sommers RK: Contributions of the fundamental, resolved harmonics, and unresolved harmonics in tonephoneme identification. J Speech Hear Res 1992;35:1406-1409.
7 Whalen DH, Xu Y: Information for Mandarin tones in the amplitude contour and in brief segments. Phonetica 1992;49:25-47.

8 Fu Q-J, Zeng FG, Shannon RV, Soli SD: Importance of tonal envelope cues in Chinese speech recognition. J Acoust Soc Am 1998; 104:505-510.

9 Liu TC, Hsu CJ, Horng MJ: Tone detection in Mandarin-speaking hearing-impaired subjects. Audiology 2000;39:106-109.

10 Schum DJ: Speech understanding in background noise; in Valente $M$ (ed): Hearing Aids: Standards, Options, and Limitations. New York, Thieme, 1996, pp 368-406.

11 Versfeld NJ, Festen JM, Houtgast T: Preference judgments of artificial processed and hearing-aid transduced speech. J Acoust Soc Am 1999;106:1566-1578.

12 American National Standards Institute: Specification of Hearing Aid Characteristics, ANSI S3.22-1996. New York, American National Standards Institute, 1996.

13 American Speech-Language-Hearing Association: Guidelines for identification audiometry. ASHA 1985;27:49-52.
14 Zhang H, Zhao KL, Wang ZZ: MACC: Chinese Minimal Auditory Capability Test. Beijing Union Hospital, 1988.

15 American National Standards Institute: Criteria for permissible ambient noise during audiometric testing, ANSI S3.1-1977. New York, American National Standards Institute, 1977.

16 Ciocca V, Francis AL, Aisha R, Wong L: The perception of Cantonese lexical tones by early-deafened cochlear implantees. J Acoust Soc Am 2002;111:2250-2256.

17 Luo X, Fu Q-J: Contribution of low-frequency acoustic information to Chinese speech recognition in cochlear implant simulations. J Acoust Soc Am 2006;120:2260-2266.

18 Borden GJ, Harris KS, Raphael LJ: Speech Science Primer: Physiology, Acoustics and Perception of Speech, ed 4. Philadelphia, Lippincott Williams \& Wilkins, 2003. 